\title{
Reciprocal repulsions instruct the precise assembly of parallel hippocampal networks
}

Daniel T. Pederick ${ }^{1}$, Jan H. Lui ${ }^{1}$, Ellen C. Gingrich ${ }^{1,2}$, Chuanyun $\mathrm{Xu}^{1}$, Mark J. Wagner ${ }^{1}$, Yuanyuan Liu $^{3, \dagger}$, Zhigang $\mathrm{He}^{3}$, Stephen R. Quake ${ }^{4,5}$, Liqun Luo ${ }^{1, *}$

${ }^{1}$ Department of Biology, Howard Hughes Medical Institute, Stanford University, Stanford, CA, USA

${ }^{2}$ Neurosciences Graduate Program, Stanford University, Stanford, CA, USA

${ }^{3}$ F.M. Kirby Neurobiology Center, Department of Neurology, Boston Children's Hospital, Harvard Medical School, Boston, MA, USA

${ }^{4}$ Departments of Bioengineering and Applied Physics, Stanford University, Stanford, CA, USA

${ }^{5}$ Chan Zuckerberg Biohub, Stanford, CA, USA

${ }^{\dagger}$ Current address: Somatosensation and Pain Unit, National Institute of Dental and Craniofacial Research (NIDCR), National Center for Complementary and Integrative Health (NCCIH), National Institutes of Health, Bethesda, MD, USA

*Corresponding author (email: 1luo@stanford.edu)

\begin{abstract}
:
Parallel information processing is a salient feature of complex nervous systems. For example, the medial and lateral hippocampal networks (MHN and LHN) preferentially process spatial- and object-related information, respectively. However, the mechanisms underlying parallel network assembly during development remain largely unknown. Here, we show that complementary expression of cell-surface molecules Teneurin-3 (Ten3) and Latrophilin-2 (Lphn2) in the MHN and LHN, respectively, guides the precise assembly of both the MHN and LHN. Viral-genetic perturbations in vivo demonstrate that Ten3+ axons are repelled by target-derived Lphn2, revealing that Lphn2/Ten3-mediated repulsion and Ten3/Ten3-mediated attraction cooperate to control precise target selection of MHN axons. In the LHN, Lphn2+ axons are confined to Lphn2+ targets via repulsion from Ten3+ targets. Our findings demonstrate that assembly of parallel hippocampal networks follows a 'Ten3 $\rightarrow$ Ten3, Lphn2 $\rightarrow$ Lphn2' rule instructed by reciprocal repulsions.
\end{abstract}




\section{Introduction}

Parallel information processing is a salient feature of complex nervous systems, enabling animals to simultaneously process diverse environmental stimuli and efficiently orchestrate appropriate actions. This is achieved through neural networks containing information-processing streams organized in parallel. In order for information to be accurately processed, neural networks must be properly assembled. While remarkable progress has been made in determining how axons are guided to the appropriate anatomical region and select specific target neurons within the region (Kolodkin and Tessier-Lavigne, 2011; Sanes and Zipursky, 2020), these mechanisms have largely been investigated in invertebrate circuits and relatively simple vertebrate circuits owing to their technical ease, with a focus on individual connections. Much less is known about how target selection is achieved in complex networks in the mammalian brain, which contains abundant parallel and often reciprocal connections across multiple regions.

One such parallel organization is present within the hippocampal-entorhinal complex, which is critical for explicit memory and spatial representation (Hafting et al., 2005; O'Keefe and Dostrovsky, 1971; Scoville and Milner, 2000; Squire et al., 2004). Spatial- and object-related information is preferentially processed by two anatomically adjacent parallel networks, the medial and lateral hippocampal networks (MHN and LHN), respectively (Cembrowski et al., 2018; Igarashi et al., 2014). The MHN and LHN consist of topographic projections along the proximal-distal axis between CA1, subiculum, and entorhinal cortex. Specifically, proximal CA1 (pCA1) projects axons to distal subiculum (dSub), while distal CA1 (dCA1) projects axons to proximal subiculum (pSub). Both pCA1 and dSub also form reciprocal connections with the medial entorhinal cortex (MEC), forming the MHN. In parallel, both $\mathrm{dCA} 1$ and pSub form reciprocal connections with the lateral entorhinal cortex (LEC), forming the LHN (Naber et al., 2001; Tamamaki and Nojyo, 1995) (Figure 1A).

We have previously shown that the type II transmembrane protein Tenuerin-3 (Ten3) has matching expression in all interconnected regions of the MHN (Figure S1A). Ten3 is required in both pCA1 and $\mathrm{dSub}$ for the precise target selection of the $\mathrm{pCA} 1 \rightarrow \mathrm{dSub}$ axons, and promotes aggregation of non-adhesive cells (Berns et al., 2018). These data support a homophilic attraction mechanism by which Ten3 regulates the pCA $1 \rightarrow \mathrm{dSub}$ axon projection in the precise assembly of the MHN. It remains unclear whether matching gene expression exists in the LHN and how this contributes to parallel hippocampal network assembly. Does the assembly of the LHN utilize a mechanism similar to or distinct from that of the MHN? Are the assemblies of MHN and LHN coordinated?

\section{Results}

\section{Complementary Lphn2/Ten3 expression across parallel hippocampal networks}

We hypothesized that cell-surface molecules that are preferentially expressed in the LHN and thus have inverse expression to Ten3 may play a role in the precise assembly of parallel hippocampal networks. To identify such molecules, we performed fluorescence-activated cell sorting-based single-cell RNA sequencing (scRNA-seq) of postnatal day 8 (P8) excitatory neurons, in MHN subregions (pCA1, dSub, and MEC) or LHN subregions (dCA1, pSub, and LEC) (see figs. S1, S2, and table S1 for details). We used the 'ground-truth' that Ten3 has enriched expression in all MHN subregions (Berns et al., 2018) to 
specifically search for genes with inverse expression patterns to Ten3 in CA1, subiculum, and entorhinal cortex. We identified Lphn2, an adhesion G-protein-coupled receptor (GPCR) known to bind Teneurins (Boucard et al., 2014; Li et al., 2018; O’Sullivan et al., 2012; Sando et al., 2019; Silva et al., 2011; del Toro et al., 2020), as one of the few cell-surface molecules that showed inverse expression to Ten3 in CA1, subiculum, and entorhinal cortex (Figure 1B and table S1). Teneurins are known to interact in trans with Latrophilins, and this interaction has been implicated in multiple neurodevelopmental processes (Sando et al., 2019; del Toro et al., 2020; Vysokov et al., 2018). This finding suggests that Lphn2 may participate in guiding the assembly of hippocampal networks.

To validate the finding from our scRNA-seq screen, we performed in situ expression analysis on P8 brains. Double in situ hybridization for Lphn 2 and Ten 3 mRNA revealed specific expression of Lphn2 in dCA1, pSub, and LEC, which was complementary to Ten3 enrichment in pCA1, dSub, and MEC (Figure 1C and Figure S3A). Quantification of Lphn2 and Ten3 mRNA showed opposing gradients of expression, with Lphn2 expression levels increasing more sharply after the Ten3-high zone in CA1 and subiculum compared to entorhinal cortex (Figure 1D and Figure S3B). We also examined protein expression using an anti-Ten3 antibody (Berns et al., 2018) and an anti-GFP antibody in Lphn2-mVenusknockin mice (Anderson et al., 2017). In all regions, Lphn2 and Ten3 proteins were expressed in the synaptic layers corresponding to their mRNA expression, including molecular layer of CA1, cell body and molecular layers of the subiculum, and layer III of the entorhinal cortex (Figure 1E and F and Figure S3C and D).

The hippocampal networks include extended projections from the subiculum to the anteroventral thalamus and the medial mammillary nucleus (Ishizuka, 2001; Meibach and Siegel, 1977; Witter and Groenewegen, 1990; Wright et al., 2010, 2013). These projections also display targeting specificity within the medial and lateral hippocampal networks and can be highlighted by tracing from the Lphn2+ and Ten3+ regions of the subiculum (Figure S4). Double in situ hybridization for Lphn2 and Ten3 mRNA in subiculum target regions of P8 mice revealed that Lphn2 and Ten3 expression matched axonal projections from neurons in the Lphn2+ and Ten3+ subiculum regions. Lphn2 showed enriched expression in the posteromedial portion of the anteroventral thalamus and the medial part of the medial mammillary nucleus, coinciding with axon projection targets of proximal, Lphn2+ subiculum neurons. By contrast, Ten3 was highly expressed in the anterolateral portion of the anteroventral thalamus and the lateral part of the medial mammillary nucleus, coinciding with axon projection targets of distal, Ten3+ subiculum neurons (compare Figure 1G with Figure S4C and D). Expression of Lphn2 and Ten3 protein was consistent with mRNA expression in extended hippocampus target regions (Figure $1 \mathrm{G}$ and $\mathrm{H}$ ).

In summary, Lphn2 and Ten3 mRNA, as well as Lphn2 and Ten3 proteins, exhibit striking complementary expression in multiple regions of the developing extended hippocampal networks, including CA1, subiculum, entorhinal cortex, anteroventral thalamus, and medial mammillary nucleus. In all cases, the connection specificity follows a 'Ten3 $\rightarrow$ Ten3, Lphn2 $\rightarrow$ Lphn2' rule that correlates cell surface molecule expression with connectivity (Figure 1I). 


\section{Subiculum Lphn2 repels Ten3+ CA1 axons}

Ten3 directs targeting of pCA1 $\rightarrow \mathrm{dSub}$ axons in the MHN through matching expression and homophilic attraction (Berns et al., 2018). Does Lphn2 also mediate homophilic attraction to assemble the LHN? To test this, we performed an in vitro cell aggregation assay using non-adhesive K562 cells. We found that Ten3-expressing K562 cells formed aggregates as previously reported (Berns et al., 2018), but Lphn2expressing cells did not (Figure S5A and B). However, Ten3-expressing cells aggregated with Lphn2expressing cells (Figure S5A and B), consistent with the previously reported heterophilic interaction between Teneurins and Latrophilins (Anderson et al., 2017; Berns et al., 2018; Boucard et al., 2014; Li et al., 2018; del Toro et al., 2020). The heterophilic interaction of Ten3 and Lphn2 combined with their complementary expression in the MHN versus LHN suggests that the interaction between Lphn2 and Ten3 may result in repulsion, which could allow distinct target selection of the MHN and LHN.

To determine if target-derived Lphn2 repels Ten3+ axons, we interrogated the CA $1 \rightarrow$ Sub connection. Ten3+ pCA1 and Lphn2+ mid-CA1 (mCA1) axons extend along a tract above the subiculum cell body layer until they reach Ten3 $+\mathrm{dSub}$ and Lphn2+ mid-subiculum (mSub), respectively, where they invade the cell body layer of the subiculum to form synapses (Berns et al., 2018) (Figure 2A). Because the $\mathrm{CA} 1 \rightarrow$ Sub projection develops postnatally (Berns et al., 2018), we injected lentivirus (LV) expressing GFP (control) or GFP-P2A-Lphn2 into the Lphn2-low dSub of mice at P0 to create a region of subiculum expressing Lphn2 across the entire proximal-distal axis. We then injected adeno-associated virus expressing membrane-bound mCherry $(A A V-C h R 2-m C h)$ into pCA1 in these same mice as adults to label and trace Ten3+CA1 axons (Figure 2B). The portion of subiculum transduced by LV was only a subset of the total pCA1 axon targeting region along the orthogonal medial-lateral axis, allowing us to determine if pCA1 axons target LV-transduced subiculum regions differently compared to neighboring non-LVtransduced subiculum regions. In $L V$-GFP controls, pCA1 axon targeting was unaffected by GFP expression (Figure 2C and Figure S6A and B). By contrast, in $L V$-GFP-P2A-Lphn2 injected brains, pCA1 axons avoided regions where Lphn2 was misexpressed in dSub (Figure 2E and Figure S6D, E).

To further assess how Ten3+ axons target the subiculum in regions where Lphn2 is misexpressed, we generated 'mountain-plots,' where pCA1 axons (mCh) and LV injection site (GFP) intensities were plotted on the same graph as height and color, respectively. Expression of GFP alone did not affect the intensity of pCA1 axons targeting the subiculum (Figure 2D and Figure S6C). However, pCA1 axon intensity was markedly reduced in dSub regions misexpressing Lphn2 (Figure $2 \mathrm{~F}$ and Figure S6F; quantified in Figure 2K). These data suggest that Ten3+ axons are repelled by misexpressed Lphn2 at the dSub target.

\section{Repulsion requires Lphn2/Teneurin but not Lphn2/FLRT interaction}

To test whether Lphn2-mediated repulsion requires Lphn2/Ten3 interaction, we utilized a deletion of the lectin binding domain in Latrophilins, which has been shown to abolish Teneurin binding without affecting interactions with other known partners or surface expression (Boucard et al., 2014; Li et al., 2018; Sando et al., 2019; del Toro et al., 2020). We validated in our K562 cell aggregation assay that Lphn2_ALec disrupted Ten3 interaction without affecting interaction with FLRT2 (Figure S5C and D), a member of the fibronectin leucine-rich transmembrane protein family known to bind Latrophilins 
(Jackson et al., 2015; O'Sullivan et al., 2012). We then misexpressed Lphn2_ALec in subiculum to determine if pCA1 axon avoidance depends on a Lphn2/Teneurin interaction. We found that in $L V$-GFPP2A-Lphn2__Lec injected brains, Ten3+ pCA1 axons were not affected by Lphn2_ALec expression in $\mathrm{dSub}$ and displayed normal targeting to dSub (Figure 2G and Figure S7A and B). Mountain plot analysis also indicated that misexpressed Lphn2_ $\Delta$ Lec did not repel Ten3+ pCA1 axons (Figure $2 \mathrm{H}$ and K; Figure $\mathrm{S} 7 \mathrm{C})$.

Recently, FLRTs have been shown to coincidently interact with Teneurin and Latrophilin to direct synapse specificity and repulsive guidance for migrating neurons (Del Toro et al., 2020; Sando et al., 2019). Intriguingly, expression of FLRT2 was enriched in Ten3-high CA1 cells (Figure S8A), suggesting that it may play a role in pCA1 axon repulsion from Lphn2. Mutation of four residues in the olfactomedin domain of Latrophilin to alanines has been show to abolish FLRT-Lphn binding while maintaining Teneurin binding and surface expression ( $\mathrm{Lu}$ et al., 2015). We validated that in K562 cells, Lphn2_4A disrupted FLRT2 binding without affecting Ten3 binding (Figure S5E and F). Yet, overexpression of Lphn2_4A in the subiculum still caused a decrease of pCA1 axon intensity in GFP+ dSub regions compared to adjacent GFP- dSub regions (Figure 2I and Figure S7D and E) to the same extent as wildtype Lphn2 (Figure 2K). These gain-of-function experiments suggest that repulsion of Ten3+ pCA1 axons by target-derived Lphn2 requires Lphn2/Teneurin but not Lphn2/FLRT interaction.

\section{Ten3+ CA1 axons invade Lphn2-null subiculum targets}

To determine if endogenous Lphn 2 in the subiculum target is necessary for correct pCA $1 \rightarrow \mathrm{dSub}$ targeting, we performed a loss-of-function experiment by injecting $L V$-GFP-Cre into the subiculum of control and $L p h n 2^{f l f l}$ mice (Anderson et al., 2017) at P0, followed by $A A V$-ChR2-mCh in pCA1 of the same mice as

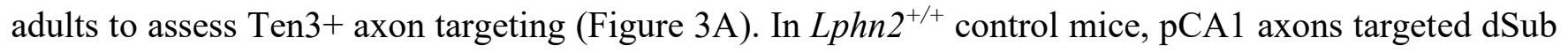
and were not disrupted when projecting into GFP-Cre+ expressing regions (Figure 3B). By contrast, Ten3+ pCA1 axons spread into the pSub of GFP-Cre+ regions in Lphn $2^{f l f l}$ mice (Figure 3C). Quantification of pCA1 axon intensity in GFP-Cre+ sections revealed that pCA1 axons in Lphn2 $2^{f l f l}$ mice had increased intensity in the more proximal regions and decreased intensity in the most distal region of the subiculum compared to Lphn $2^{+/+}$mice (Figure 3E and F; red vs black). These data suggest that Lphn2 in pSub normally repels Ten3+ pCA1 axons, enabling them to specifically target dSub.

To rule out the possibility that the ectopic invasion of pCA1 axons into $L p h n 2^{--}$pSub results from loss of Lphn2 interaction with a molecule other than Ten3 [e.g., another Teneurin that is expressed in pCA1 (Figure S8B)], we performed the same Lphn2 loss-of-function experiment in Ten3 $3^{-/}$mice. Anterograde tracing from pCA1 in $L p h n 2^{+/+} ; T e n 3^{-/-}$mice showed that pCA1 axons spread more along the proximal-distal axis of the subiculum as previously reported (Berns et al., 2018) (Figure S9A). In Lphn $2^{f l f l}$;Ten $3^{-/-}$mice, pCA1 axons also showed similar spreading (Figure S9B). We observed no significant increase of axon intensity in the proximal subiculum of $L p h n 2^{f l f l}$;Ten $3^{-/}$mice compared with Lphn $2^{+/+} ;$Ten $^{-/-}$mice (Figure S9C and D). The lack of an additional axon mistargeting phenotype in Lphn $2^{f l f l}$;Ten $3^{-/-}$mice compared to Lphn $2^{+/+} ;$Ten $3^{-/}$mice suggests that Ten 3 is required for the effect of loss of subiculum Lphn2 on pCA1 axon targeting, and that Lphn2/Ten3-mediated repulsion instructs precise $\mathrm{pCA} 1 \rightarrow \mathrm{dSub}$ target selection. 


\section{Lphn2/Ten3-mediated repulsion and Ten3/Ten3-mediated attraction cooperate}

Loss of Lphn2/Ten3 heterophilic repulsion (above) or Ten3 homophilic attraction (Berns et al., 2018) alone both disrupt precise $\mathrm{pCA} 1 \rightarrow \mathrm{dSub}$ axon targeting. What is the relative contribution of each? To test this, we simultaneously conditionally deleted both Lphn2 and Ten3 in the subiculum and assessed the targeting of Ten3+ pCA1 axons. We found that pCA1 axons projecting into GFP-Cre+ regions of $L p h n 2^{f l f l}$;Ten $3^{f l f l}$ mice targeted more proximal regions of subiculum and also had decreased fluorescence intensity in dSub (Figure 3D). Quantification of pCA1 axons in GFP-Cre+ subiculum sections of $L p h n 2^{f l f l}$;Ten $3^{f l f l}$ mice showed a significant increase in axon intensity into the $L p h n 2^{-/-}$pSub region compared to $L p h n 2^{+/+}$;Ten $3^{+/+}$mice (Figure 3E and F; blue vs black) and additionally had decreased fluorescence intensity in the Ten $3^{-/}$dSub region compared to $L p h n 2^{f l f l}$;Ten $3^{+/+}$mice (Figure $3 \mathrm{E}$ and F; blue vs red).

The increase of pCA1 axon intensity in the pSub of Lphn2 $2^{f l f l}$;Ten $3^{f l f l}$ compared to Lphn $2^{+/+} ;$Ten $^{+/+}$confirms the loss of repulsion of pCA1 axons from Lphn2+ pSub. At the same time, the decrease of axon intensity in dSub of $L p h n 2^{f l f l l}$;Ten $3^{f l f l}$ compared to $L p h n 2^{f l f l}$;Ten $3^{+/+}$indicates a loss of attraction of Ten3 + pCA1 axons to Ten3 + dSub. Thus, Lphn2/Ten3-mediated heterophilic repulsion and Ten3/Ten3-mediated homophilic attraction cooperate in orchestrating the precise targeting of pCA1 axons to dSub.

\section{Subiculum Ten3 repels Lphn2+ CA1 axons}

In addition to serving as a repulsive ligand for target selection of Ten3+ MHN neurons, could Lphn2 also act as a receptor to regulate target selection of LHN neurons? Specifically, could Lphn2+ axons be repelled from Ten3+ targets to regulate the precision of LHN connections? To test this, we injected LV-GFP-Cre into the subiculum of $T e n 3^{+/+}$(control) and Ten $3^{\text {flflf }}$ mice at P0, followed by $A A V-C h R 2-m C h$ in mCA1 of the same mice as adults to assess Lphn2+ mCA1 axon targeting (Figure 4A). In Ten3 ${ }^{+/+}$mice, mCA1 axons predominantly projected to $\mathrm{mSub}$ (Figure 4B). However, in Ten $3^{f l f l}$ mice, mCA1 axons spread into Ten3-null dSub (Figure 4C). Quantification of axons in the subiculum showed a significant increase in axon intensity in the dSub of Ten $3^{\text {flfl }}$ mice compared to Ten $3^{+/+}$mice (Figure 4D and E). Thus, Ten3 in dSub prevents Lphn2+ mCA1 axon invasion into dSub.

To test if Lphn2 in mCA1 axons is required for their target precision, we deleted Lphn2 from CA1 followed by tracing of Lphn2-null mCA1 axons (Figure 4F). Control mCA1 axons targeted mSub (Figure 4G), whereas Lphn2-null mCA1 axons spread into the most distal subiculum (Figure 4H, quantified in Figure 4I and J). Thus, Lphn2 is cell-autonomously required in mCA1 neurons to prevent their axons from invading Ten3 $+\mathrm{dSub}$. Taken together with the Ten3 conditional deletion in subiculum above, these data indicate that Lphn2+ mCA1 axons are repelled by target-derived Ten3. 


\section{Discussion}

Here, we provide multiple lines of evidence demonstrating that parallel hippocampal networks are assembled through reciprocal repulsions between Ten3- and Lphn2-expressing cells. Our results demonstrate that Lphn2 and Ten3 instruct the assembly of both MHN and LHN connections from CA1 to the subiculum (Figure 5A). Specifically, in the MHN, Lphn2/Ten3-mediated heterophilic repulsion and Ten3/Ten3-mediated homophilic attraction cooperate to instruct pCA1 $\rightarrow$ dSub targeting; in the LHN, Ten3/Lphn2-mediated heterophilic repulsion confines Lphn2+ axons to the Lphn2+ target region, contributing to the precision of $\mathrm{mCA} 1 \rightarrow \mathrm{mSub}$ targeting (Figure 5B). Together, these data show that the mechanisms required for parallel network assembly in the hippocampus are intertwined, utilizing multiple interactions of two cell-surface molecules and reciprocal repulsions to ensure precise segregated connections are formed.

Our results have expanded our understanding of the roles of Lphn2 by showing that it acts both cell non-autonomously in targets and cell autonomously in axons during the target selection stage of hippocampal circuit assembly, preceding synapse formation. This is in contrast to previous studies suggesting that Latrophilins act strictly as postsynaptic adhesion molecules to establish or maintain synapses (Anderson et al., 2017; Sando et al., 2019; Südhof, 2018). While defects in axon targeting may contribute to previously reported findings of synaptic deficits in Latrophilin early postnatal loss-offunction experiments (Anderson et al., 2017; Sando et al., 2019), our study does not rule out the possibility that Latrophilin/Teneurin interactions play additional roles in synaptic adhesion if the repulsive mechanism is switched off after target selection is complete. Latrophilins bind both Teneurins and FLRTs, and the cooperative binding of these three proteins has been implicated in directing synapse specificity and repulsion-mediated neuron migration (Del Toro et al., 2020; Sando et al., 2019). However, misexpression of a mutant Lphn2 that cannot bind FLRT (Figure S5E and F) still repelled Ten3+ pCA1 axons to the same extent (Figure 2K), suggesting that FLRT binding is not required for Lphn2/Ten3mediated repulsion during target selection of axons.

We identify Ten3/Lphn2 as a membrane-bound repulsive ligand/receptor pair during circuit assembly, joining the likes of Ephrin/Eph, Dscam/Dscam, and clustered protocadherin/protocadherin (Cheng et al., 1995; Drescher et al., 1995; Lefebvre et al., 2012; Matthews et al., 2007). The mechanisms of how an extracellular adhesive interaction leads to repulsion are poorly understood. In vitro studies have suggested that proteolytic cleavage (Drescher et al., 1995) or endocytosis (Hattori et al., 2000; Zimmer et al., 2003) of the receptor complex may be involved in allowing repulsion after Ephrin/Eph extracellular interactions. Both Latrophilins and Teneurins have been shown to undergo cleavage of their extracellular domains (Araç et al., 2012; Vysokov et al., 2018). It is conceivable that Lphn2-Ten3 binding, while sending a repulsive signal to Ten3+ or Lphn2+ growth cones, also triggers the cleavage of Ten3 and/or Lphn2, thus terminating a transient axon-target interaction.

Our results highlight the cooperation of repulsion and attraction and multifunctionality of cellsurface proteins during target selection. Cooperation of attraction and repulsion has been described at different stages of neuronal circuit assembly (Kolodkin and Tessier-Lavigne, 2011; Sanes and Yamagata, 2009). For example, the PlexB receptor interacts with Sema2a and Sema2b through repulsion and attraction, respectively, to mediate distinct guidance functions during Drosophila sensory circuit assembly 
(Wu et al., 2011). We found that target selection of pCA1 axons is determined by Lphn2/Ten3-mediated repulsion from $\mathrm{pSub}$ and Ten3/Ten3-mediated attraction to dSub (Figure 3). Thus, Ten3 acts as a receptor for both repulsive and attractive ligands in the same axon during target selection. Conversely, Ten3 acts as a ligand as an attractant for Ten3+ axons, but a repellent for Lphn2+ axons (Figure 5).

A striking finding in this study is the complementary expression of Ten 3 and Lphn 2 across all interconnected regions of the hippocampal network (Figure 1I). This is reminiscent of Ephrin-A/EphA counter-gradients found across interconnected regions of the developing visual system (Lambot et al., 2005) that utilize bi-directional Ephrin-A/EphA interactions for the formation of topographic projections (Cang et al., 2008; Egea and Klein, 2007; Feldheim et al., 2000, 2004; Frisén et al., 1998; Rashid et al., 2005). The patterns of Ten 3 and Lphn 2 expression across the hippocampal network follow a 'Ten3 $\rightarrow$ Ten3, Lphn2 $\rightarrow$ Lphn2' rule (Figure 1I). We have demonstrated that target-derived Lphn2 acts as a repulsive ligand for Ten3+ axons in the MHN, and that target-derived Ten3 acts as a repulsive ligand for Lphn2+ axons in the LHN. These reciprocal repulsions may guide target selection across seven additional projections of the MHN and LHN that follow the 'Ten3 $\rightarrow$ Ten3, Lphn2 $\rightarrow$ Lphn2' rule. It will be interesting to determine if complementary expression of cell-surface molecules and reciprocal repulsions are used in additional parallel networks across the brain.

The repeated use of the same molecules to guide target selection across extended networks, together with the multifunctionality of a single protein serving as receptor and ligand, contribute to explaining how a limited number of cell-surface molecules can specify a much larger number of connections in the mammalian brain. 


\section{References:}

Anders, S., Pyl, P.T., and Huber, W. (2015). HTSeq--a Python framework to work with high-throughput sequencing data. Bioinformatics 31, 166-169.

Anderson, G.R., Maxeiner, S., Sando, R., Tsetsenis, T., Malenka, R.C., and Südhof, T.C. (2017). Postsynaptic adhesion GPCR latrophilin-2 mediates target recognition in entorhinal-hippocampal synapse assembly. J. Cell Biol. 216, 3831-3846.

Araç, D., Boucard, A.A., Bolliger, M.F., Nguyen, J., Soltis, S.M., Südhof, T.C., and Brunger, A.T. (2012). A novel evolutionarily conserved domain of cell-adhesion GPCRs mediates autoproteolysis. EMBO J 31, 1364-1378.

Berns, D.S., DeNardo, L.A., Pederick, D.T., and Luo, L. (2018). Teneurin-3 controls topographic circuit assembly in the hippocampus. Nature 554, 328-333.

Boucard, A.A., Maxeiner, S., and Südhof, T.C. (2014). Latrophilins function as heterophilic cell-adhesion molecules by binding to teneurins: regulation by alternative splicing. J. Biol. Chem. 289, 387-402.

Butler, A., Hoffman, P., Smibert, P., Papalexi, E., and Satija, R. (2018). Integrating single-cell transcriptomic data across different conditions, technologies, and species. Nature Biotechnology 36, 411-420.

Cang, J., Wang, L., Stryker, M.P., and Feldheim, D.A. (2008). Roles of ephrin-as and structured activity in the development of functional maps in the superior colliculus. J Neurosci 28, 11015-11023.

Cembrowski, M.S., Phillips, M.G., DiLisio, S.F., Shields, B.C., Winnubst, J., Chandrashekar, J., Bas, E., and Spruston, N. (2018). Dissociable Structural and Functional Hippocampal Outputs via Distinct Subiculum Cell Classes. Cell 173, 12801292.e18.

Cheng, H.J., Nakamoto, M., Bergemann, A.D., and Flanagan, J.G. (1995). Complementary gradients in expression and binding of ELF-1 and Mek4 in development of the topographic retinotectal projection map. Cell 82, 371-381.

Del Toro, D., Carrasquero-Ordaz, M.A., Chu, A., Ruff, T., Shahin, M., Jackson, V.A., Chavent, M., Berbeira-Santana, M., Seyit-Bremer, G., Brignani, S., et al. (2020). Structural Basis of Teneurin-Latrophilin Interaction in Repulsive Guidance of Migrating Neurons. Cell 180, 323-339.e19.

Dobin, A., Davis, C.A., Schlesinger, F., Drenkow, J., Zaleski, C., Jha, S., Batut, P., Chaisson, M., and Gingeras, T.R. (2013). STAR: ultrafast universal RNA-seq aligner. Bioinformatics 29, 15-21.

Drescher, U., Kremoser, C., Handwerker, C., Löschinger, J., Noda, M., and Bonhoeffer, F. (1995). In vitro guidance of retinal ganglion cell axons by RAGS, a $25 \mathrm{kDa}$ tectal protein related to ligands for Eph receptor tyrosine kinases. Cell 82, 359-370.

Egea, J., and Klein, R. (2007). Bidirectional Eph-ephrin signaling during axon guidance. Trends Cell Biol. 17, $230-238$.

Feldheim, D.A., Kim, Y.I., Bergemann, A.D., Frisén, J., Barbacid, M., and Flanagan, J.G. (2000). Genetic analysis of ephrinA2 and ephrin-A5 shows their requirement in multiple aspects of retinocollicular mapping. Neuron 25, 563-574.

Feldheim, D.A., Nakamoto, M., Osterfield, M., Gale, N.W., DeChiara, T.M., Rohatgi, R., Yancopoulos, G.D., and Flanagan, J.G. (2004). Loss-of-function analysis of EphA receptors in retinotectal mapping. J Neurosci 24, 2542-2550.

Frisén, J., Yates, P.A., McLaughlin, T., Friedman, G.C., O’Leary, D.D., and Barbacid, M. (1998). Ephrin-A5 (AL-1/RAGS) is essential for proper retinal axon guidance and topographic mapping in the mammalian visual system. Neuron 20, $235-243$.

Hafting, T., Fyhn, M., Molden, S., Moser, M.-B., and Moser, E.I. (2005). Microstructure of a spatial map in the entorhinal cortex. Nature 436, 801-806. 
Harris, J.A., Hirokawa, K.E., Sorensen, S.A., Gu, H., Mills, M., Ng, L.L., Bohn, P., Mortrud, M., Ouellette, B., Kidney, J., et al. (2014). Anatomical characterization of Cre driver mice for neural circuit mapping and manipulation. Front Neural Circuits 8,76 .

Hattori, M., Osterfield, M., and Flanagan, J.G. (2000). Regulated cleavage of a contact-mediated axon repellent. Science 289, $1360-1365$.

Igarashi, K.M., Ito, H.T., Moser, E.I., and Moser, M.-B. (2014). Functional diversity along the transverse axis of hippocampal area CA1. FEBS Letters 588, 2470-2476.

Ishizuka, N. (2001). Laminar organization of the pyramidal cell layer of the subiculum in the rat. J. Comp. Neurol. 435, 89110 .

Jackson, V.A., del Toro, D., Carrasquero, M., Roversi, P., Harlos, K., Klein, R., and Seiradake, E. (2015). Structural Basis of Latrophilin-FLRT Interaction. Structure 23, 774-781.

Kinoshita, M., Matsui, R., Kato, S., Hasegawa, T., Kasahara, H., Isa, K., Watakabe, A., Yamamori, T., Nishimura, Y., Alstermark, B., et al. (2012). Genetic dissection of the circuit for hand dexterity in primates. Nature 487, $235-238$.

Kolodkin, A.L., and Tessier-Lavigne, M. (2011). Mechanisms and molecules of neuronal wiring: a primer. Cold Spring Harb Perspect Biol 3.

Lambot, M.-A., Depasse, F., Noel, J.-C., and Vanderhaeghen, P. (2005). Mapping labels in the human developing visual system and the evolution of binocular vision. J. Neurosci. 25, 7232-7237.

Leamey, C.A., Merlin, S., Lattouf, P., Sawatari, A., Zhou, X., Demel, N., Glendining, K.A., Oohashi, T., Sur, M., and Fässler, R. (2007). Ten_m3 regulates eye-specific patterning in the mammalian visual pathway and is required for binocular vision. PLoS Biol. 5, e241.

Lefebvre, J.L., Kostadinov, D., Chen, W.V., Maniatis, T., and Sanes, J.R. (2012). PROTOCADHERINS MEDIATE DENDRITIC SELF-AVOIDANCE IN THE MAMMALIAN NERVOUS SYSTEM. Nature 488, 517-521.

Lein, E.S., Hawrylycz, M.J., Ao, N., Ayres, M., Bensinger, A., Bernard, A., Boe, A.F., Boguski, M.S., Brockway, K.S., Byrnes, E.J., et al. (2007). Genome-wide atlas of gene expression in the adult mouse brain. Nature 445, 168-176.

Li, J., Shalev-Benami, M., Sando, R., Jiang, X., Kibrom, A., Wang, J., Leon, K., Katanski, C., Nazarko, O., Lu, Y.C., et al. (2018). Structural Basis for Teneurin Function in Circuit-Wiring: A Toxin Motif at the Synapse. Cell 173, 735-748.e15.

Lu, Y.C., Nazarko, O.V., Sando, R., Salzman, G.S., Li, N.-S., Südhof, T.C., and Araç, D. (2015). Structural Basis of Latrophilin-FLRT-UNC5 Interaction in Cell Adhesion. Structure 23, 1678-1691.

Madisen, L., Zwingman, T.A., Sunkin, S.M., Oh, S.W., Zariwala, H.A., Gu, H., Ng, L.L., Palmiter, R.D., Hawrylycz, M.J., Jones, A.R., et al. (2010). A robust and high-throughput Cre reporting and characterization system for the whole mouse brain. Nat. Neurosci. 13, 133-140.

Matthews, B.J., Kim, M.E., Flanagan, J.J., Hattori, D., Clemens, J.C., Zipursky, S.L., and Grueber, W.B. (2007). Dendrite self-avoidance is controlled by Dscam. Cell 129, 593-604.

Meibach, R.C., and Siegel, A. (1977). Efferent connections of the septal area in the rat: an analysis utilizing retrograde and anterograde transport methods. Brain Res. 119, 1-20.

Naber, P.A., Lopes da Silva, F.H., and Witter, M.P. (2001). Reciprocal connections between the entorhinal cortex and hippocampal fields CA1 and the subiculum are in register with the projections from CA1 to the subiculum. Hippocampus 11 , 99-104. 
O’Keefe, J., and Dostrovsky, J. (1971). The hippocampus as a spatial map. Preliminary evidence from unit activity in the freely-moving rat. Brain Res 34, 171-175.

O’Sullivan, M.L., de Wit, J., Savas, J.N., Comoletti, D., Otto-Hitt, S., Yates, J.R., and Ghosh, A. (2012). FLRT proteins are endogenous latrophilin ligands and regulate excitatory synapse development. Neuron 73, 903-910.

Picelli, S., Faridani, O.R., Björklund, A.K., Winberg, G., Sagasser, S., and Sandberg, R. (2014). Full-length RNA-seq from single cells using Smart-seq2. Nat Protoc 9, 171-181.

Rashid, T., Upton, A.L., Blentic, A., Ciossek, T., Knöll, B., Thompson, I.D., and Drescher, U. (2005). Opposing gradients of ephrin-As and EphA7 in the superior colliculus are essential for topographic mapping in the mammalian visual system.

Neuron 47, 57-69.

Sando, R., Jiang, X., and Südhof, T.C. (2019). Latrophilin GPCRs direct synapse specificity by coincident binding of FLRTs and teneurins. Science 363 .

Sanes, J.R., and Yamagata, M. (2009). Many Paths to Synaptic Specificity. Annual Review of Cell and Developmental Biology 25, 161-195.

Sanes, J.R., and Zipursky, S.L. (2020). Synaptic Specificity, Recognition Molecules, and Assembly of Neural Circuits. Cell $181,536-556$.

Scoville, W.B., and Milner, B. (2000). Loss of recent memory after bilateral hippocampal lesions. 1957. J Neuropsychiatry Clin Neurosci 12, 103-113.

Seiradake, E., del Toro, D., Nagel, D., Cop, F., Härtl, R., Ruff, T., Seyit-Bremer, G., Harlos, K., Border, E.C., Acker-Palmer, A., et al. (2014). FLRT Structure: Balancing Repulsion and Cell Adhesion in Cortical and Vascular Development. Neuron $84,370-385$.

Silva, J.-P., Lelianova, V.G., Ermolyuk, Y.S., Vysokov, N., Hitchen, P.G., Berninghausen, O., Rahman, M.A., Zangrandi, A., Fidalgo, S., Tonevitsky, A.G., et al. (2011). Latrophilin 1 and its endogenous ligand Lasso/teneurin-2 form a high-affinity transsynaptic receptor pair with signaling capabilities. Proc Natl Acad Sci U S A 108, 12113-12118.

Squire, L.R., Stark, C.E.L., and Clark, R.E. (2004). The medial temporal lobe. Annu Rev Neurosci 27, 279-306.

Südhof, T.C. (2018). Towards an Understanding of Synapse Formation. Neuron 100, 276-293.

Tamamaki, N., and Nojyo, Y. (1995). Preservation of topography in the connections between the subiculum, field CA1, and the entorhinal cortex in rats. J. Comp. Neurol. 353, 379-390.

del Toro, D., Carrasquero-Ordaz, M.A., Chu, A., Ruff, T., Shahin, M., Jackson, V.A., Chavent, M., Berbeira-Santana, M., Seyit-Bremer, G., Brignani, S., et al. (2020). Structural Basis of Teneurin-Latrophilin Interaction in Repulsive Guidance of Migrating Neurons. Cell 180, 323-339.e19.

Vysokov, N.V., Silva, J.-P., Lelianova, V.G., Suckling, J., Cassidy, J., Blackburn, J.K., Yankova, N., Djamgoz, M.B., Kozlov, S.V., Tonevitsky, A.G., et al. (2018). Proteolytically released Lasso/teneurin-2 induces axonal attraction by interacting with latrophilin-1 on axonal growth cones. ELife 7, e37935.

Weissbourd, B., Ren, J., DeLoach, K.E., Guenthner, C.J., Miyamichi, K., and Luo, L. (2014). Presynaptic partners of dorsal raphe serotonergic and GABAergic neurons. Neuron 83, 645-662.

Witter, M.P., and Groenewegen, H.J. (1990). The subiculum: cytoarchitectonically a simple structure, but hodologically complex. Progress in Brain Research 83, 47-58. 
Wright, N.F., Erichsen, J.T., Vann, S.D., O’Mara, S.M., and Aggleton, J.P. (2010). Parallel but separate inputs from limbic cortices to the mammillary bodies and anterior thalamic nuclei in the rat. J. Comp. Neurol. 518, 2334-2354.

Wright, N.F., Vann, S.D., Erichsen, J.T., O’Mara, S.M., and Aggleton, J.P. (2013). Segregation of parallel inputs to the anteromedial and anteroventral thalamic nuclei of the rat. J. Comp. Neurol. 521, 2966-2986.

Wu, Z., Sweeney, L.B., Ayoob, J.C., Chak, K., Andreone, B.J., Ohyama, T., Kerr, R., Luo, L., Zlatic, M., and Kolodkin, A.L. (2011). A Combinatorial Semaphorin Code Instructs the Initial Steps of Sensory Circuit Assembly in the Drosophila CNS. Neuron 70, 281-298.

Zimmer, M., Palmer, A., Köhler, J., and Klein, R. (2003). EphB-ephrinB bi-directional endocytosis terminates adhesion allowing contact mediated repulsion. Nat. Cell Biol. 5, 869-878.

\section{Acknowledgements:}

We thank T. Südhof for the Lphn $2^{m V e n u s}$ and $L p h n 2^{f l}$ mice, the Neuroscience Gene Vector and Virus Core at Stanford University for producing viruses, D. Berns for advice, inspiration, and artwork (Figure 1a, i), J. Ferguson for artwork (Figure $2 \mathrm{a}$ and Figure 4k), J. Kebschull for custom MATLAB code, H. Meng for virus preparation, members of the Luo lab for advice and support, D. Berns, J. Kebschull, A. Khalaj, H. Li, J. Li, T. Li, C. McLaughlin, K. Shen and A. Shuster for critiques of the manuscript.

\section{Funding:}

D.T.P. was supported by an American Australian Association Education Fund Scholarship. L.L. is an investigator of Howard Hughes Medical Institute. This work was supported by National Institutes of Health grant (R01-NS050580 to L.L.).

\section{Author contributions:}

D.T.P. performed all the experiments and analyzed the data, except for single-cell sequencing sample collection and data processing which was performed by J.H.L., with support from S.R.Q. E.C.G. and C.X. assisted in tissue processing. M. J. W. generated MATLAB code and analyzed data. Y.L. and Z.H. produced custom lentivirus. L.L. supervised the study. D.T.P., J.H.L., and L.L. wrote the paper.

\section{Competing interests:}

The authors declare no competing interests.

\section{Supplementary Materials:}

Materials and Methods

Figures S1-S10

Tables S1

Additional references 

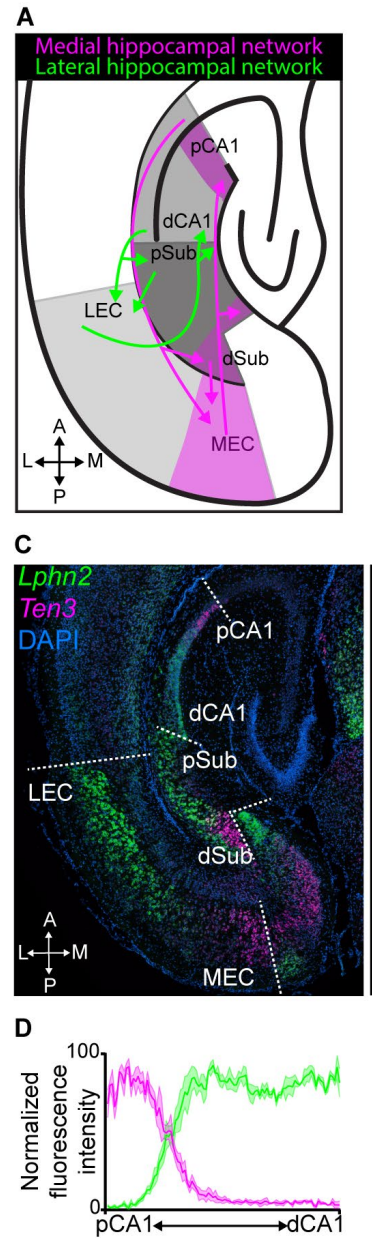

E

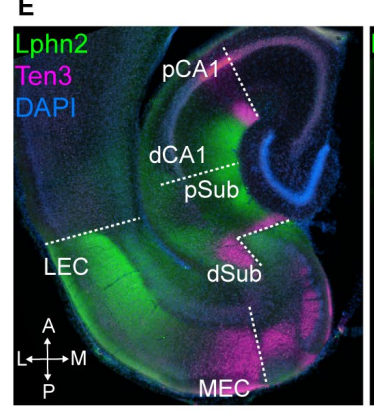

F

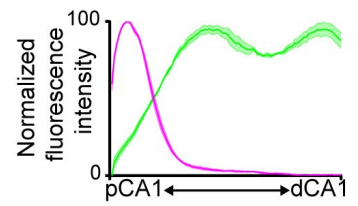

B
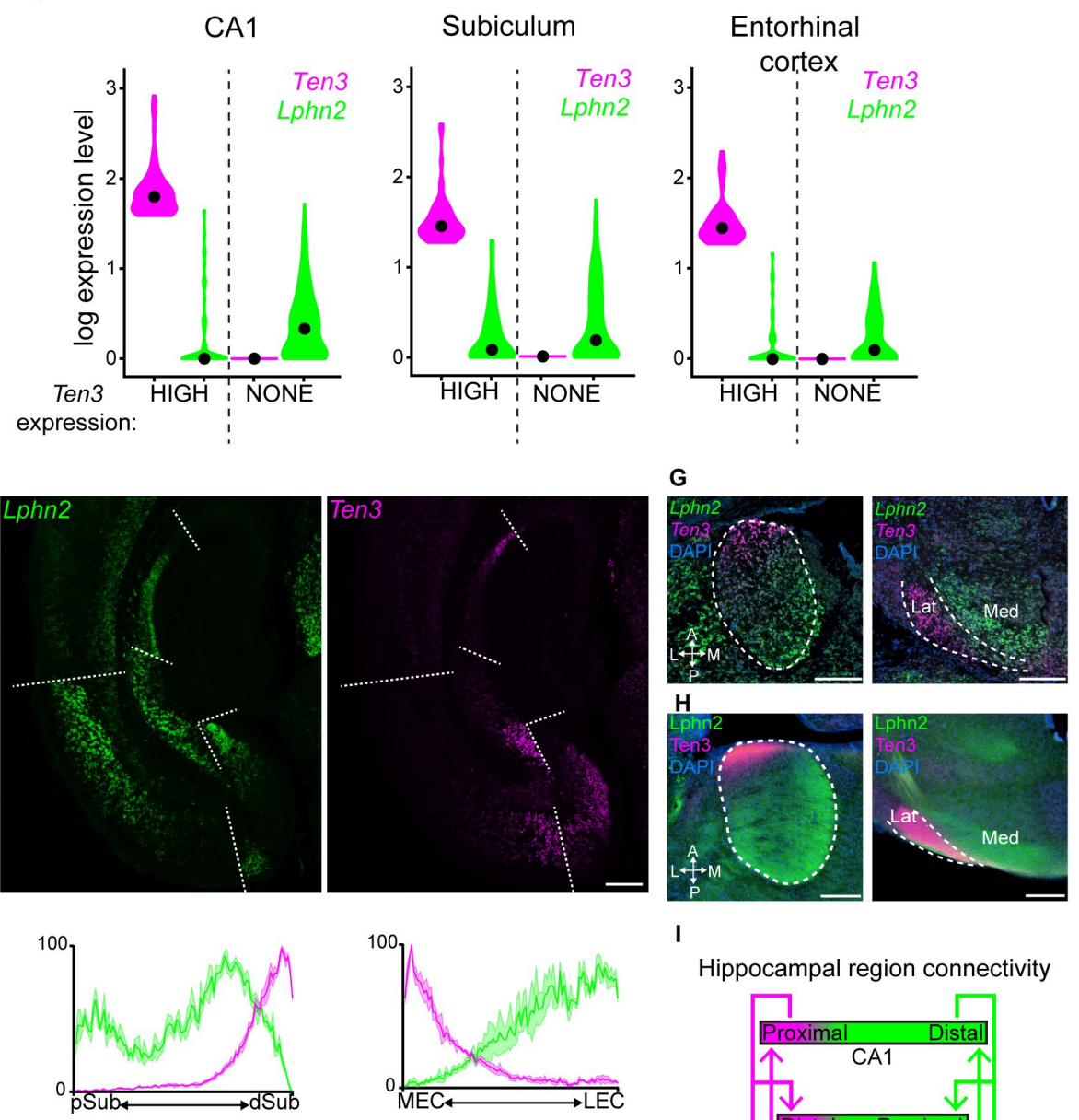

I

Hippocampal region connectivity

'Extended hippocampus' connectivity
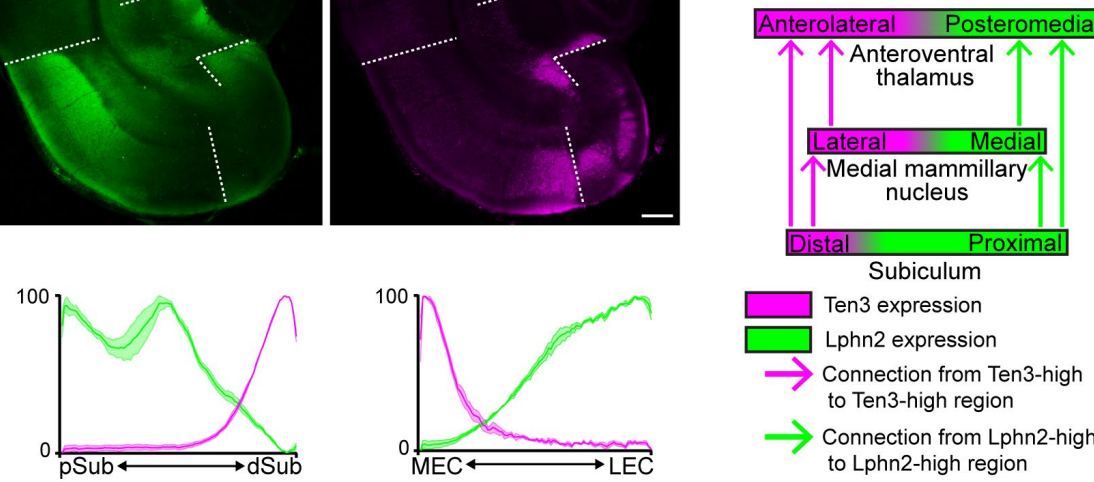
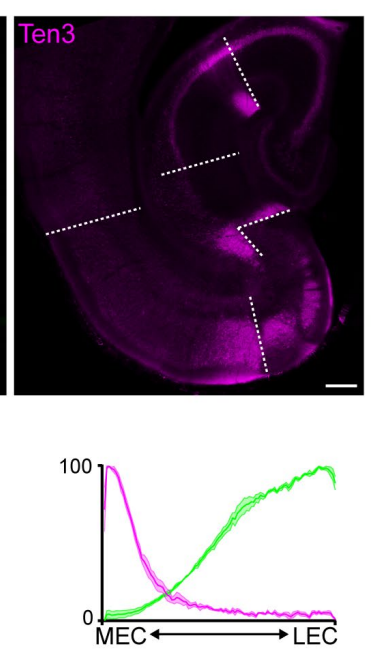
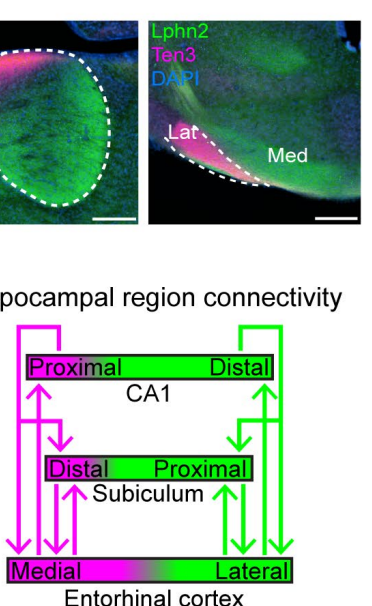

Subiculum Ten3 expression Lphn2 expression to Ten3-high region

$\rightarrow$ Connection from Lphn2-high to Lphn2-high region $\rightarrow$ Connection from Ten3-high

Figure 1. Complementary expression patterns of Lphn2 and Ten3 in the hippocampal network. (A) Summary of axonal projection patterns of medial (magenta) and lateral (green) hippocampal network connections. Magenta regions have high Ten3 expression (Berns et al., 2018). A, anterior; P, posterior: L, lateral; M, medial. (B) Violin plots highlighting Lphn2 and Ten3 expression in Ten3-HIGH and Ten3NONE cells across CA1, subiculum, and entorhinal cortex. The unit of expression level is $\ln [1+$ (reads 
per 10000)]. Black dots represent the median. (C) Double in situ hybridization for Lphn2 (middle) and Ten3 (right) mRNA on a horizontal section of P8 mouse brain. Dashed lines represent boundaries between CA1, subiculum, and entorhinal cortex as labeled in the overlay (left). (D) Quantification of Lphn2 and Ten 3 mRNA across the proximal-distal axis of CA1 $(n=3$ mice, 29 sections total $)$ and subiculum $(n=3$ mice, 31 sections total) cell body layers and the medial-lateral axis of layer III entorhinal cortex $(n=3$ mice, 22 sections total). Mean \pm SEM. (E) Double immunostaining for Lphn2 (middle; anti-GFP antibody) and Ten3 (right) on a horizontal section of P8 Lphn2-mVenus knock-in mouse (Anderson et al., 2017) brain. Dashed lines represent boundaries between CA1, subiculum, and entorhinal cortex as labeled in the overlay (left). (F) Quantification of Lphn2 and Ten3 protein across the proximal-distal axis of molecular layers of CA1 ( $n=3$ mice, 23 sections total) and subiculum $(n=3$ mice, 22 sections total), and the mediallateral axis of layer III entorhinal cortex ( $n=3$ mice, 14 sections total). Mean \pm SEM. (G) Double in situ hybridization showing Lphn2 and Ten3 mRNA expression in the anteroventral thalamus (left) and medial mammillary nucleus (right) of a P8 mouse. Lat: lateral; Med; medial. (H) Double immunostaining for Lphn2 (left; anti-GFP antibody) and Ten3 on a P8 Lphn2-mVenus knock-in mouse (Anderson et al., 2017) brain, showing protein expression in anteroventral thalamus (left) and the medial mammillary nucleus (right). Dashed lines represent anteroventral nucleus (left) and the boundary between the medial and lateral regions of the medial mammillary nucleus (right) in both $(\mathbf{G})$ and $(\mathbf{H})$. (I) Schematic summary of the expression pattern of Lphn2 and Ten3 in relation to interconnected regions of the lateral and medial hippocampal networks. Scale bars, $200 \mu \mathrm{m}$. 

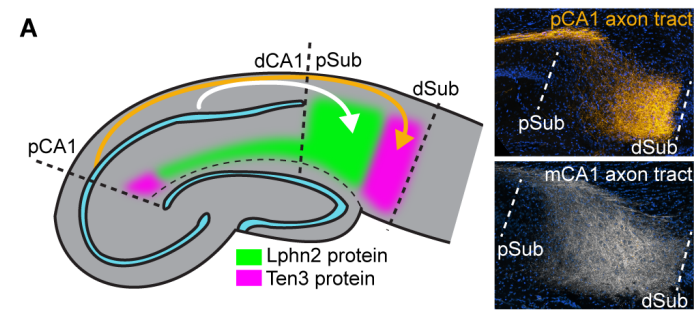

B

C

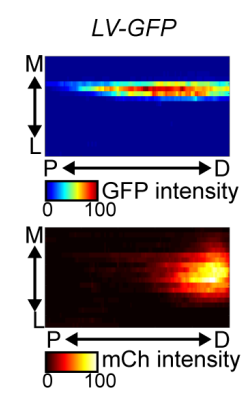

D

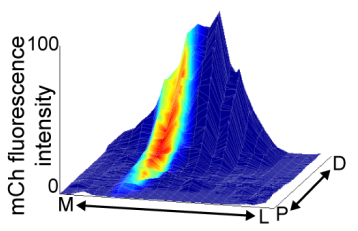

E

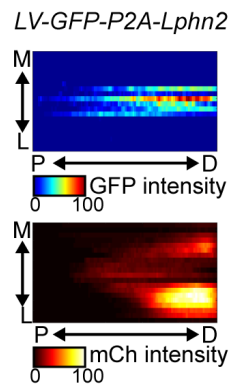

$\mathbf{F}$

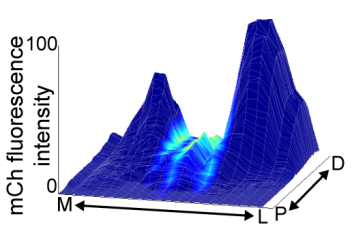

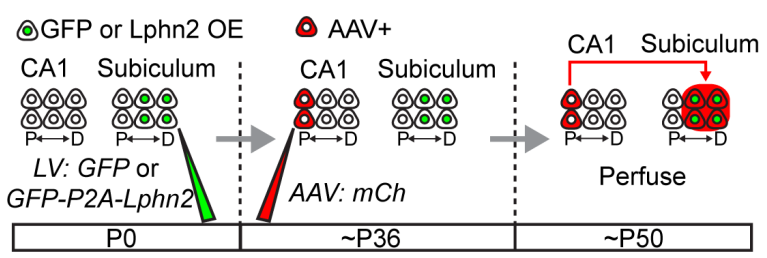

G LV-GFP-P2A-LPhn2_LLec

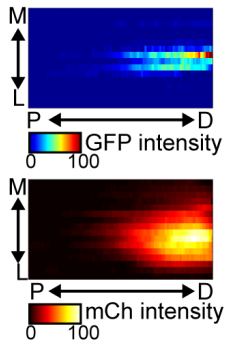

H
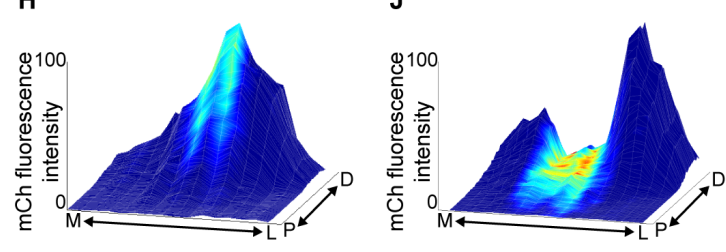

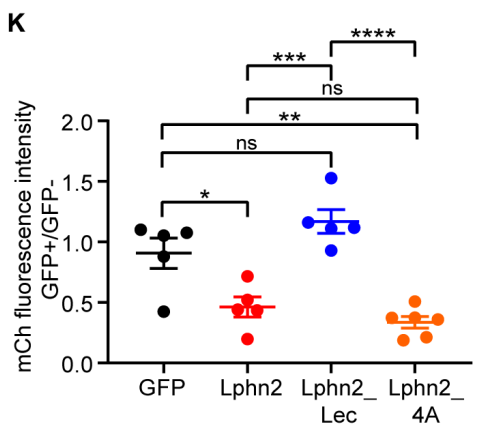

Figure 2. Ten3+ pCA1 axons avoid distal subiculum misexpressing Lphn2 in a Lphn2/Teneurin interaction-dependent and Lphn2/FLRT interaction-independent manner. (A) Left, schematic highlighting the trajectory of pCA1 (orange) and mCA1 (white) axons to the subiculum. Right, confocal images of adult subiculum showing pCA1 axons (orange, top) and mCA1 (white, bottom) extending along a tract above the subiculum cell body layer until they turn into dSub and mSub target areas, respectively. (B) Experimental design. (C), (E), (G), (I) Heatmaps showing normalized GFP fluorescence intensity (top) and normalized $\mathrm{mCh}$ fluorescence intensity (bottom) in subiculum. Each row is one section; adjacent sections are separated by $120 \mu \mathrm{m}$. Lentiviral (LV) constructs injected are above each pair of panels. P, proximal; D, distal; M, medial; L, lateral. (D), (F), (H), (J) Mountain-plots showing normalized GFP fluorescence intensity as color and normalized $\mathrm{mCh}$ fluorescence intensity as height. Same data as $(\mathbf{C})$, (E), (G), (I), respectively. (K) Ratio of mCh fluorescent intensity of GFP+ versus GFP- regions. $L V$-GFP 
bioRxiv preprint doi: https://doi.org/10.1101/2020.05.28.122242; this version posted February 24, 2021. The copyright holder for this preprint

(which was not certified by peer review) is the author/funder, who has granted bioRxiv a license to display the preprint in perpetuity. It is made available under aCC-BY-NC-ND 4.0 International license.

$(\mathrm{n}=5), L V-G F P-P 2 A-L p h n 2(\mathrm{n}=5), L V-G F P-P 2 A-L p h n 2 \_\Delta L e c(\mathrm{n}=5)$ and $L V-G F P-P 2 A-L p h n 2 \_4 A(\mathrm{n}$ $=6$ ). Mean \pm SEM; one-way ANOVA with Tukey's multiple comparisons test. **** $P \leq 0.0001$; *** $P \leq$ $0.001 ; * * P \leq 0.01 ; * P \leq 0.05$; ns, not significant. 

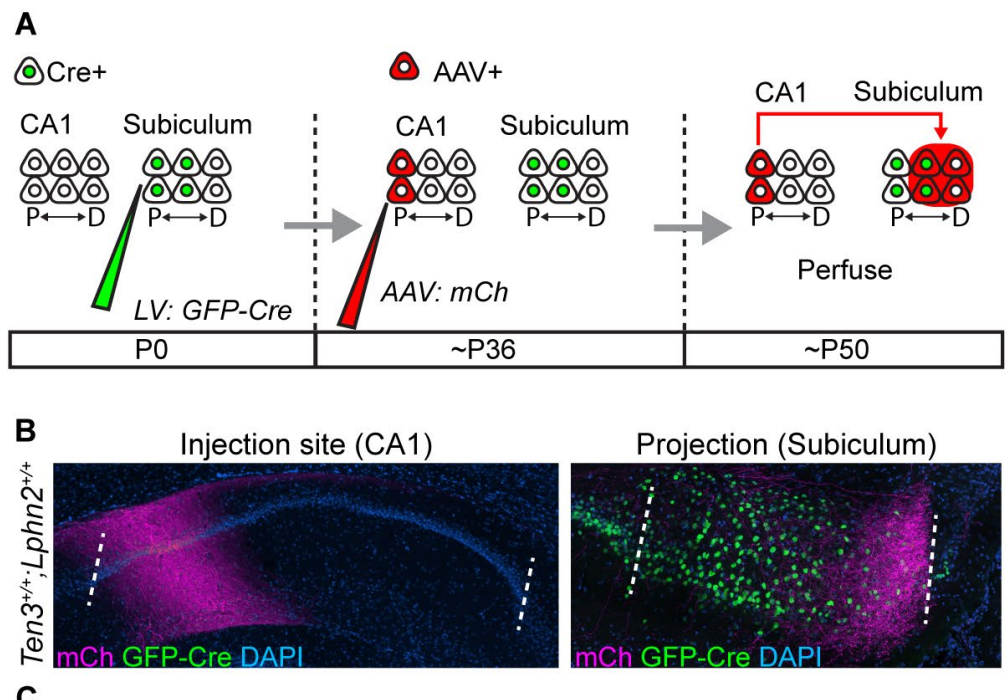

C
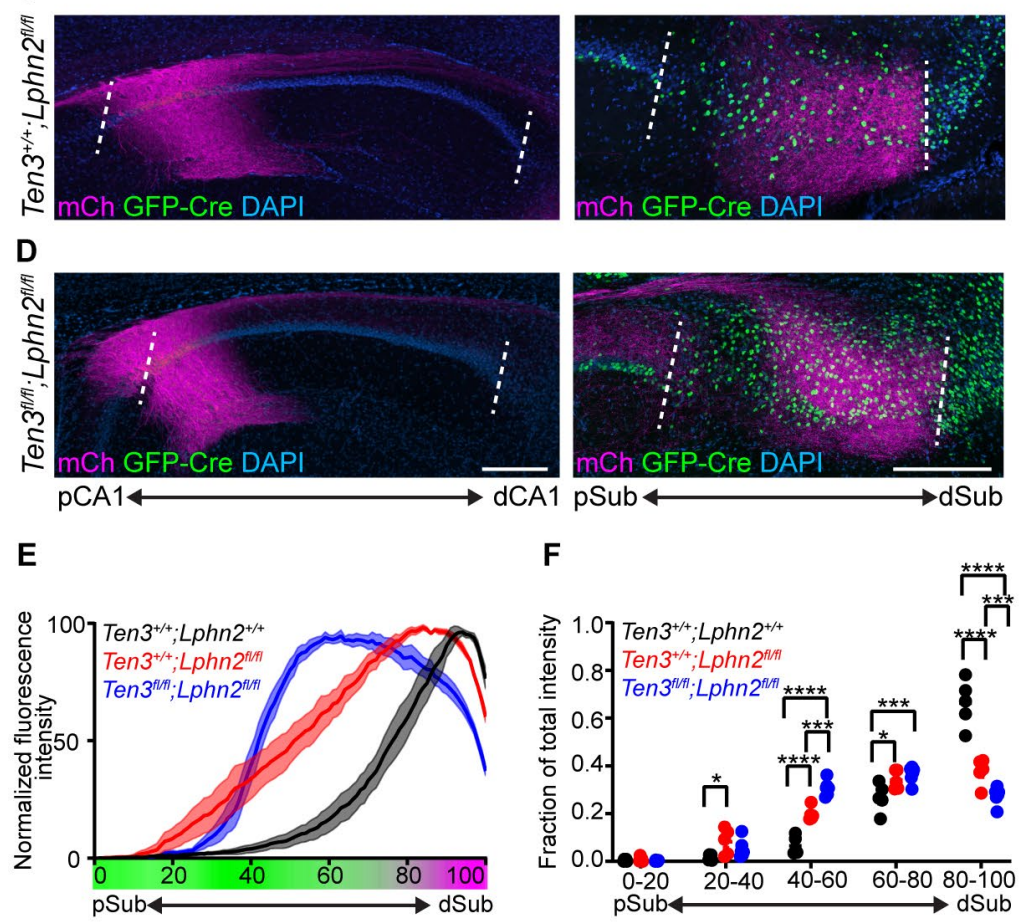

Figure 3. Lphn2/Ten3-mediated repulsion and Ten3/Ten3-mediated attraction cooperate to guide pCA1 $\rightarrow$ dSub target selection. (A) Experimental design. (B to D) Representative images of $A A V-m C h$ (magenta) injections in pCA1 (left) and corresponding projections overlapping with $L V$-GFP-Cre (green) injection sites in the subiculum (right). Genetic conditions are as indicated. (E) Normalized mean fluorescence intensity traces of subiculum projections from pCA1 in GFP-Cre+ sections for Lphn2 ${ }^{+/+}(\mathrm{n}$ $=5$ mice), Lphn $2^{f l f l}(\mathrm{n}=5$ mice $)$ and $L p h n 2^{f l f f l} ; \operatorname{Ten}^{f l / f l}(\mathrm{n}=6$ mice). Mean \pm SEM. Color bar under $\mathrm{X}$-axis represents Lphn2 (green) and Ten3 (magenta) expression in subiculum as quantified in Figure S3. (F) Fraction of total axon intensity for the same data as (E) across 20 percent intervals. Mean \pm SEM, twoway ANOVA with Sidak's multiple comparisons test. **** $P \leq 0.0001 ; * * * P \leq 0.001 ; * P \leq 0.05$. Scale bar, $200 \mu \mathrm{m}$. Injection site locations in CA1 are shown in Figure S1 

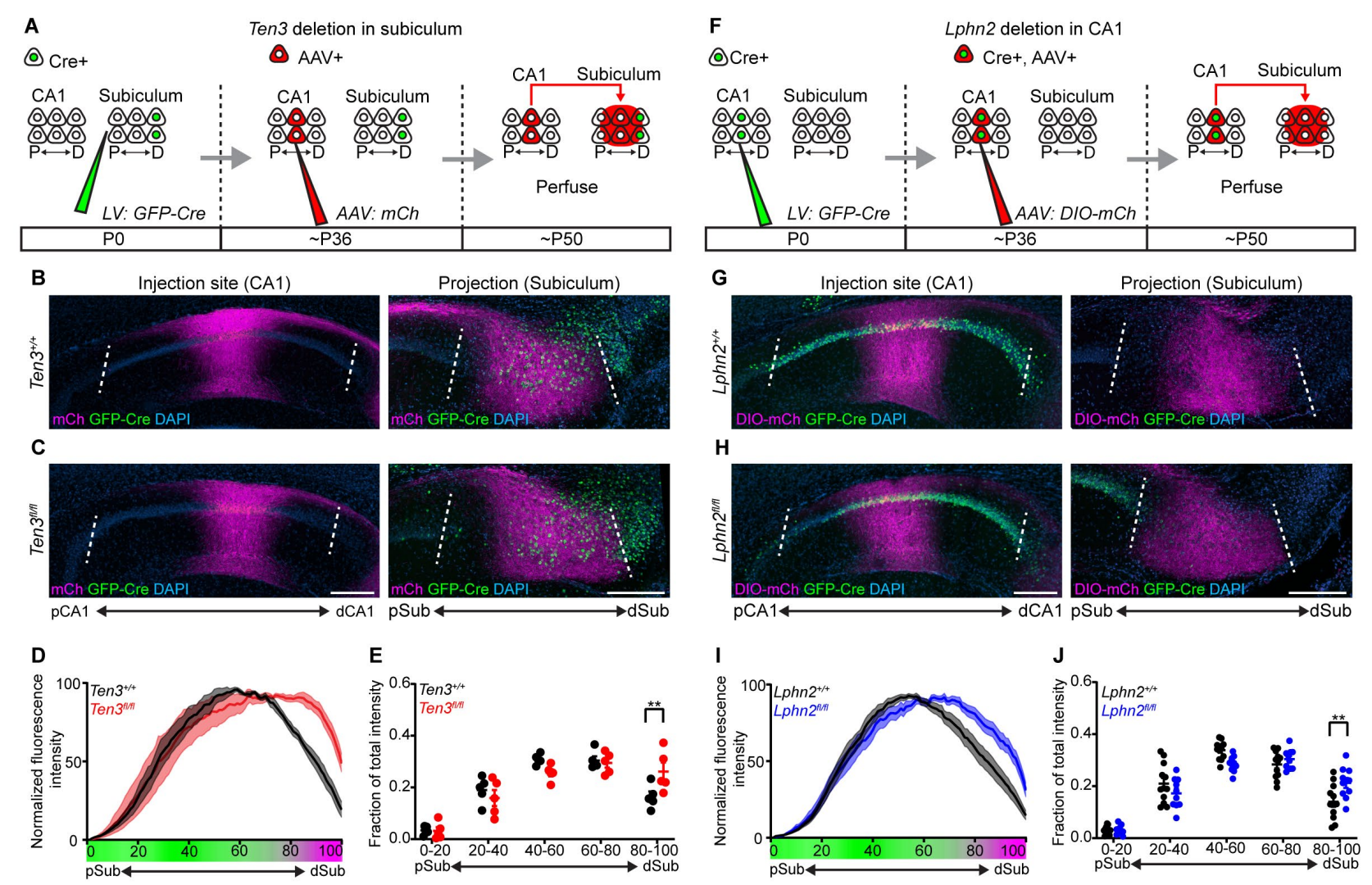

Figure 4. Lphn2+ mCA1 axons avoid Ten3+ dSub, contributing to LHN target selection. (A) Experimental design for deleting Ten3 in the subiculum and tracing mCA1 axons. (B and C) Representative images of $A A V-m C h$ (magenta) injections in mCA1 (left) and corresponding projections overlapping with $L V$-GFP-Cre (green) injection sites in the subiculum (right). Genetic conditions are as indicated. (D) Normalized mean fluorescence intensity traces of subiculum projections from mCA1 in GFP-Cre+ sections for Ten $3^{+/+}(\mathrm{n}=5$ mice $)$ and Ten $3^{f l f l}(\mathrm{n}=5$ mice $)$. Mean \pm SEM. Color bar under $\mathrm{x}-$ axis represents Lphn2 (green) and Ten3 (magenta) expression in subiculum as quantified in Figure S3. (E) Fraction of total axon intensity for the same data as (D) across 20 percent intervals. Mean \pm SEM; two-way ANOVA with Sidak's multiple comparisons test, ** $P \leq 0.01$. (F) Experimental design for deleting Lphn2 in CA1 and tracing Lphn2-null mCA1 axons. (G and $\mathbf{H})$ Representative images of $A A V$ $D I O-m C h$ (magenta) injections in mCA1 (left) and corresponding projections in the subiculum (right). Genetic conditions are as indicated. (I) Normalized mean fluorescence intensity traces of subiculum projections from mCA1 axons for $L p h n 2^{+/+}\left(\mathrm{n}=12\right.$ mice) and $L p h n 2^{f l f l}(\mathrm{n}=10$ mice). Mean $\pm \operatorname{SEM}$. Color bar under x-axis represents Lphn2 (green) and Ten3 (magenta) expression in subiculum as quantified in Figure S3. (J) Fraction of total axon intensity for the same data as (I) across 20 percent intervals. Mean \pm SEM; two-way ANOVA with Sidak's multiple comparisons test; $* * P \leq 0.01$. Scale bars, $200 \mu \mathrm{m}$. Injection site locations in CA1 are shown in Figure S10. 

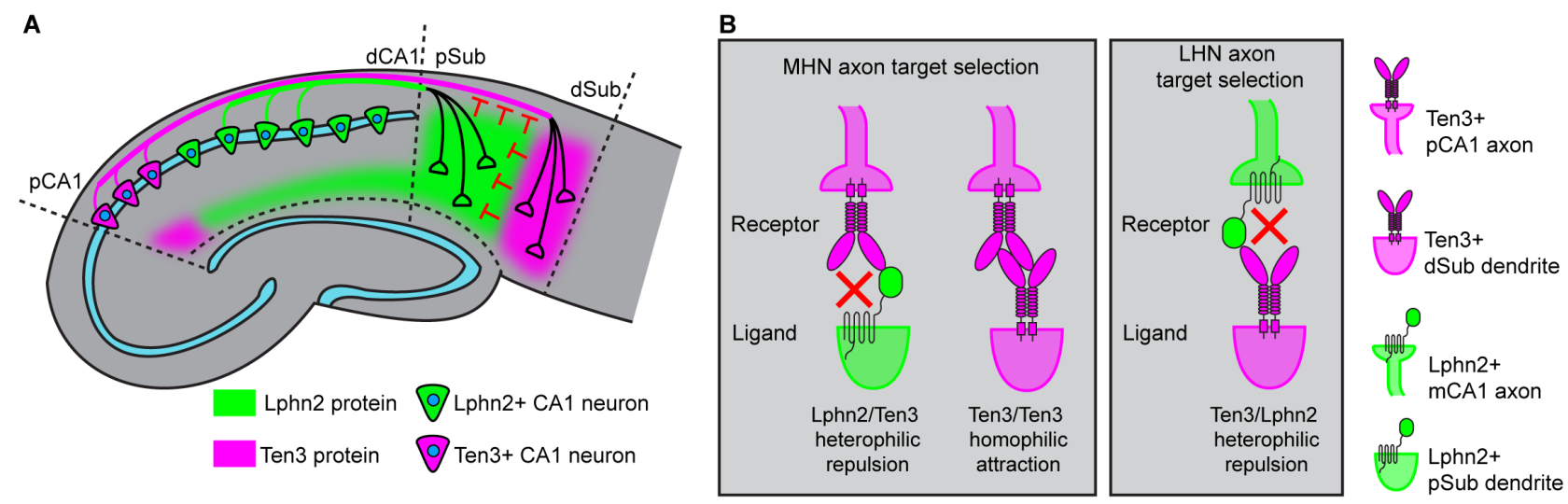

Figure 5. Lphn2 and Ten3 instruct target selection of hippocampal axons through reciprocal repulsions. (A) Ten3 + pCA1 axons target Ten3 $+\mathrm{dSub}$ via repulsion from Lphn2 and attraction to Ten3 in the subiculum. Lphn2+ mCA1 axons target Lphn2+ mSub via repulsion from Ten3 in dSub. (B) Highmagnification view of ligand-receptor interactions that instruct target selection of Ten3+ MHN (left) and Lphn2+ LHN (right) axons. Red crosses symbolize repulsion. 
bioRxiv preprint doi: https://doi.org/10.1101/2020.05.28.122242; this version posted February 24, 2021. The copyright holder for this preprint (which was not certified by peer review) is the author/funder, who has granted bioRxiv a license to display the preprint in perpetuity. It is made available under aCC-BY-NC-ND 4.0 International license. 